\title{
A novel peptide from TCTA protein inhibits proliferation of fibroblast-like synoviocytes of rheumatoid arthritis patients
}

\author{
YUKI NANKE, TORU YAGO, TSUYOSHI KOBASHIGAWA, MANABU KAWAMOTO, \\ HISASHI YAMANAKA, SHIGERU KOTAKE
}

Institute of Rheumatology, Tokyo Women's Medical University, Shinjuku-ku, Tokyo, Japan

\begin{abstract}
Background: We have demonstrated that a peptide, which we named 'Peptide A', derived from the extracellular domain of T-cell leukemia translocation-associated gene (TCTA) protein, inhibited human osteoclastogenesis.

Objective: In the current study, we examined whether this peptide inhibits the proliferation of rheumatoid arthritis (RA) fibroblast-like synoviocytes (FLS) or not.

Material and methods: Fibroblast-like synoviocytes obtained from five RA patients were cultured in the absence or presence of 1, 5, $10 \mu \mathrm{g} / \mathrm{ml}$ of peptide. We used 29-mer scrambled peptide as a control.

Results: The peptide inhibited the proliferation of RA FLS dose-dependently. On the other hand, the scrambled peptide showed no inhibition.

Conclusions: The peptide inhibits both human osteoclastogenesis and the proliferation of RA FLS. Thus, the peptide may be used for the therapy of both osteoporosis and synovitis of RA patients.

This is the first report of the new peptide we discovered, which inhibits both osteoclastogenesis and synovitis. Thus, this new peptide could be a new drug for patients with both osteoporosis and RA.
\end{abstract}

Key words: osteoclast, arthritis, synovium, translocation-associated gene (TCTA), T-cell leukemia.

(Centr Eur J Immunol 2014; 39 (4): 468-470)

\section{Introduction}

In 1995, Aplan et al. cloned and characterized a novel gene at the site of a $\mathrm{t}(1 ; 3)(\mathrm{p} 34 ; \mathrm{p} 21)$ translocation breakpoint in T-cell acute lymphoblastic leukemia, designating this gene as TCTA [1]. TCTA mRNA is expressed ubiquitously in normal tissues, with the highest levels of expression in the kidney. TCTA has been conserved throughout evolution in organisms ranging from Drosophila to humans. A short open reading frame encodes a protein of 103 amino acid residues, $M_{r}$ 11,300, without strong homology to any previously reported proteins. Of note, genomic Southern blots demonstrated a reduced TCTA signal in three of four small cell lung cancer cell lines, suggesting the loss of one of the two copies of the gene [1]. On the other hand, in 2005, it was reported that TCTA interacts with SMA- and MAD-related protein 4 (SMAD4) in a proteome-scale map of the human protein-protein interaction network (Supplementary Table
S2, line 6175 of ref. 2); however, the function of TCTA has not been clarified.

In 2009, we identified a novel peptide, which we named 'Peptide A', from TCTA protein expressed in synovial tissues of patients with rheumatoid arthritis (RA) that regulates human osteoclastogenesis [3]. In the study, we therefore purified proteins from synovial tissues of patients with RA, using gel filtration chromatography, reverse-aspect HPLC, and mass spectrometry [3]. We finally demonstrated that a peptide derived from the extracellular domain of TCTA protein inhibited both RANKL-induced human osteoclastogenesis and pit formation of mature human osteoclasts [3].

In the current study, we investigated the effect of a peptide from TCTA protein on the proliferation of FLS. The peptide significantly inhibited the proliferation of FLS. 


\section{Material and methods}

\section{Materials}

Synovial tissues were obtained from 5 patients with RA at the time of total knee replacement surgery. All patients gave their informed consent.

\section{Cell proliferation assay}

Proliferation of FLS was measured using a Cell Proliferation Assay Kit (XTT baser) (Biological Industries Ltd. Israel). The assay was performed according to the manufacturer's protocol. Cells $\left(0.6-1.0 \times 10^{3} /\right.$ well $)$ were

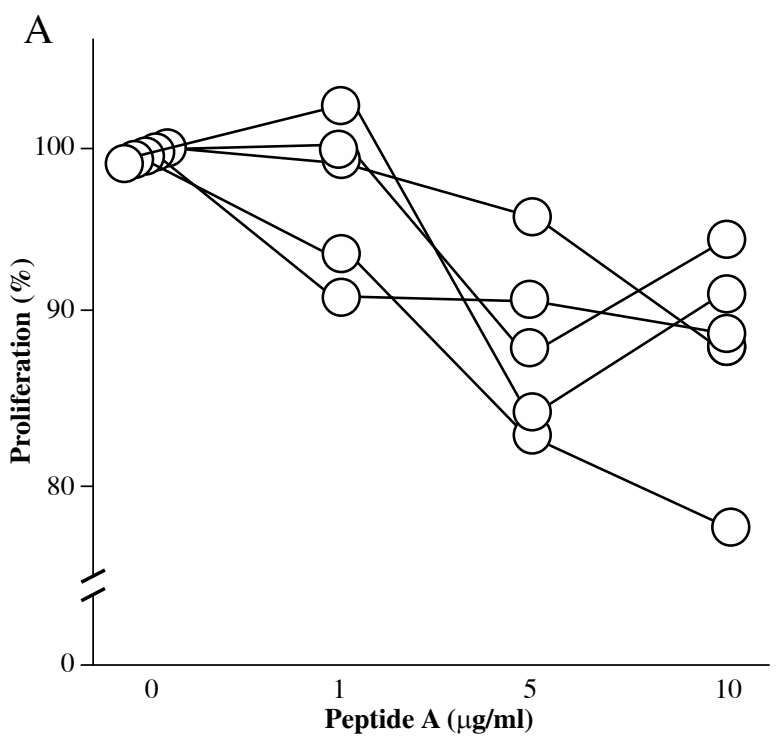

$$
\mathrm{C}
$$

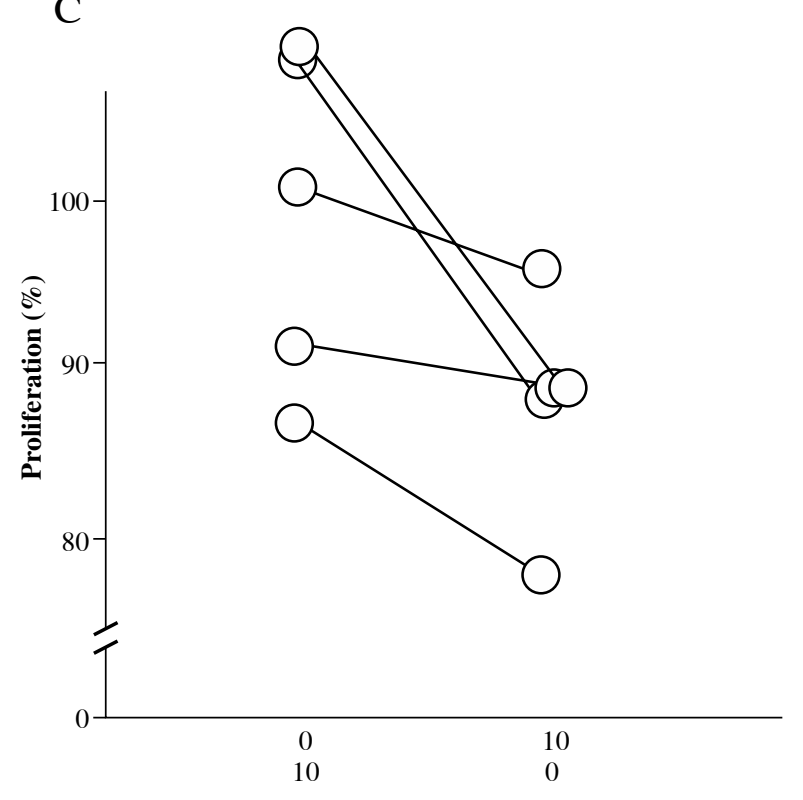

Peptide A Scrambled $(\mu \mathrm{g} / \mathrm{ml})$ cultured in 96-well plates. Before cells were confluent, various concentrations of Peptide A or scrambled peptide as a control were added to the wells. After 24 or $48 \mathrm{~h}$, the cells were collected, and cell proliferation was measured using the kit. Experiments were repeated 5 times. All experiments were performed in quadruplicate.

\section{Statistical analysis}

Significance was analyzed using the Wilcoxon's rank sum test. The results are expressed as the means $\pm \mathrm{SD}$, and considered significant at a $p$-value of $<0.05$.

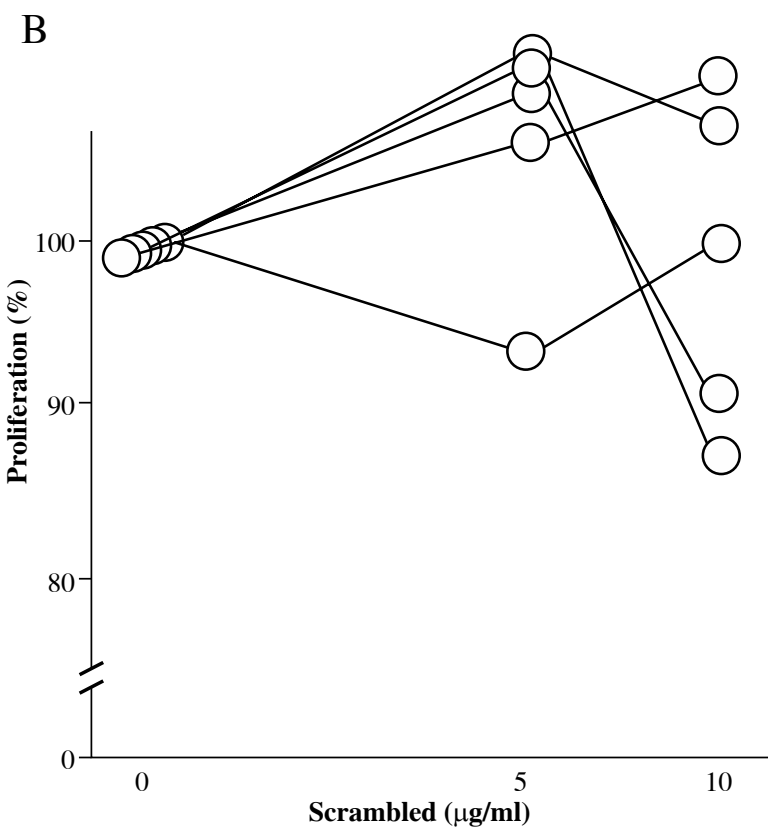

Fibroblast-like synoviocytes was cultured with various concentrations of Peptide A (A) or scrambled peptides (B). The proliferation levels with $0,1,5,10 \mu \mathrm{g} / \mathrm{ml}$ are shown for Peptide A and $0,5,10 \mu \mathrm{g} / \mathrm{ml}$ are shown for scrambled peptides, respectively. The proliferation levels with $10 \mu \mathrm{g} / \mathrm{ml}$ are compared between Peptide A and scrambled peptide in $\mathrm{C}$

Fig. 1. Cell proliferation assay 


\section{Results}

\section{Peptide A significantly inhibited the proliferation of fibroblast-like synoviocytes}

Peptide A dose-dependently inhibited the proliferation of FLS (Fig. 1A). On the other hand, the scrambled peptide used as a control did not inhibit the proliferation of FLS (Fig. 1B). At $10 \mu \mathrm{g} / \mathrm{ml}$, Peptide A significantly inhibited the proliferation of FLS compared with the scrambled peptide (Wilcoxon test, $p=0.031$, Fig. 1C).

\section{Discussion}

In the current study, we demonstrated that Peptide A dose-dependently inhibited the cell proliferation of FLS from RA patients. On the other hand, the scrambled peptide as a control did not inhibit the proliferation of FLS. We previously demonstrated that toxicity is not detected in human cells cultured with Peptide A [3]. Thus, our findings suggest that Peptide A is useful in the treatment of synovitis in RA patients, although the mechanism of the inhibition remains to be elucidated.

Peptide A may be useful for patients with osteoporosis as well, because we have demonstrated that Peptide A inhibits the formation and function of osteoclasts [3]. Osteoclastogenesis and activity of osteoclasts are triggered and/ or supported by proinflammatory cytokines overproduced in RA patients. Therefore, these patients are prone to both local and systemic osteoporosis [4]. In the current study, we demonstrated that Peptide A inhibited the proliferation of FLS. Thus, Peptide A is a novel strategy for RA patients with synovitis and osteoporosis.

We have also reported that Peptide A significantly inhibited the cell proliferation of a small-cell lung carcinoma cell line, RERF-LC-MA [5]. In addition, TCTA protein was detected in RERF-LC-MA by immunohistological staining [5]. Recently, it has been reported that breast cancer cell lines induce osteoclastogenesis in a paracrine manner [6]. Thus, the peptide may provide a novel therapeutic strategy for patients with small-cell lung carcinoma, especially with bone metastasis [5].

Interestingly, synovitis of RA patients and cancer metastasis/invasiveness have some similar behavior. For example, the regulation of cell motility, proliferation and apoptosis, as well as stimulation of angiogenesis and remodeling of the extracellular matrix are important in both pathological conditions [7]. The hallmark of metastatic activity of tumor cells and the invasive behavior of synovial fibroblasts in RA appear similar. Thus, it is intriguing that Peptide A inhibits the proliferation of both FLS and RERF-LC-MA.

In conclusion, Peptide A inhibited the cell proliferation of FLS from RA. Since Peptide A inhibits human osteoclastogenesis as we previously reported [3], Peptide A may be useful for the treatment of RA with osteoporosis.

\section{Acknowledgments}

We thank Ms. Hanae Kikuchi (Tokyo Women's Med. Univ.) for valuable technical assistance.

Ethics approval: The study received ethical approval from the Institute of Rheumatology, Tokyo Women's Medical University.

The authors declare no conflict of interest.

\section{References}

1. Aplan PD, Johnson BE, Russell E, et al. (1995): Cloning and characterization of TCTA, a gene located at the site of at $(1 ; 3)$ translocation. Cancer Res 55: 1917-1921.

2. Rual JF, Venkatesan K, Hao T, et al. (2005): Towards a proteome-scale map of the human protein-protein interaction network. Nature 437: 1173-1178.

3. Kotake S, Nanke Y, Kawamoto M, et al. (2009): T-cell leukemia translocation-associated gene (TCTA) protein is required for human osteoclastogenesis. Bone 45: 627-639.

4. Kotake S, Udagawa N, Takahashi N, et al. (1999): IL-17 in synovial fluids from patients with rheumatoid arthritis is a potent stimulator of osteoclastogenesis. J Clin Invest 103: 1345-1352.

5. Kotake S, Yago T, Kawamoto M, Nanke Y (2013): A Novel Peptide from T-cell leukemia translocation-associated gene (TCTA) protein inhibits proliferation of a small-cell lung carcinoma. J Cancer Ther 4: 44-46.

6. Costa-Rodrigues J, Moniz KA, Teixeira MR, Fernandes MH (2012): Variability of the paracrine-induced osteoclastogenesis by human breast cancer cell lines. J Cell Biochem 113: 1069-1079.

7. Oslejsková L, Grigorian M, Gay S, et al. The metastasis associated protein S100A4: a potential novel link to inflammation and consequent aggressive behaviour of rheumatoid arthritis synovial fibroblasts. Ann Rheum Dis 2008; 67: 1499-1504. 